\title{
The monobloc hydrogel breast implant, experiences and ideas
}

\author{
Rita M. Kappel • Ger J. M. Pruijn
}

Received: 18 January 2011 / Accepted: 30 April 2011 /Published online: 1 July 2011

(C) The Author(s) 2011. This article is published with open access at Springerlink.com

\begin{abstract}
This study is focused on the properties of the monobloc hydrogel (MH) breast implant, which has been around for more than 30 years, and to see how it behaves with regard to health complaints as sometimes seen in some patients who had received silicone gel (SG) breast implants. Patients responded to a questionnaire examining their experience with breast implants. Three groups were included. First, the control group $(n=34)$ of women without breast implants. Second, a C group of women $(n=42)$ who began and remained on the MH implant. Third, the B group of women who had their silicone gel implant replaced by the MH implant. In the B1 subgroup $(n=22)$, a capsulectomy was also performed. In the B2 subgroup $(n=13)$, the replacement was carried out without a capsulectomy. The $\mathrm{C}$ group behaved very much like the control group. The women of the B group experienced an improvement of their complaints and the improvement was even better after a capsulectomy. The only difference between the MH and SG implants is the content of the implant. The satisfaction of women with $\mathrm{MH}$ implants is generally high and not or hardly associated with health complaints. In women with SG implants and health complaints, these complaints can be relieved by replacement of the implants by $\mathrm{MH}$ implants.
\end{abstract}

\footnotetext{
R. M. Kappel $(\bowtie)$

Department of Plastic and Reconstructive Surgery 926, Radboud University Nijmegen Medical Center, PO Box 9101, 6500 HB Nijmegen, The Netherlands e-mail: R.Kappel@plchir.umcn.nl

G. J. M. Pruijn

Department of Biomolecular Chemistry 271, Nijmegen Center for Molecular Life Sciences, Institute for Molecules and Materials, Radboud University Nijmegen,

PO Box 9101, NL-6500 HB Nijmegen, The Netherlands
}

Keywords Monobloc hydrogel implant $\cdot$ Silicone breast implant $\cdot$ Breast augmentation · Breast reconstruction

\section{Introduction}

For decades, breast implants have been used to augment or reconstruct the female breasts. Three types of implants are available: implants filled with saline, implants filled with silicone gel (SG) and silicone oil and implants filled with a mixture of saline and cellulose, called the monobloc hydrogel (MH). The SG implant is used most frequently, whereas the saline implants are hardly used anymore. All of these breast implants have a silicone elastomere shell.

The MH breast implant was developed in the 1970s by the late Arion [1]. He was a French plastic surgeon who was also an engineer and he was motivated to create this implant by the patients who had silicone granulomas [2]. The major property of the MH implant is a biodegradable content of $97 \%$ saline and 3\% cellulose (carboxymethylcellulose). The MH implant comes very close to being a saline implant, and with this, it has in common a chance of spontaneous rupture. The reason for this is unknown. In case of a rupture, the hydrogel will cause the production of clear serous fluid, without the implant becoming empty. As a result there will be an increase of one cup size within a couple of days and therefore the patient will be alarmed by the implant itself that it has ruptured. The replacement can be planned as an elective procedure under local anaesthesia of the submammary scar within $20 \mathrm{~min}$, because the implant is never adherent to the capsule as is usually the case with a textured SG implant. The serous liquid is drained and the use of drains is a contraindication. The MH-augmented or -reconstructed breast looks very natural (Fig. 1) and feels soft because of the absence of capsular 
Fig. 1 Breast augmentation and reconstruction with monobloc hydrogel implants. Images of pre-operative (a) and post-operative (b) chest of a woman who had breast augmentation with the $\mathrm{MH}$ implant. Images of pre-operative (c) and post-operative (d) chest of a woman who had a breast reconstruction with the $\mathrm{MH}$ implant. Because of irradiation on the right side, a muscular cutaneous latissimus dorsi flap was transposed
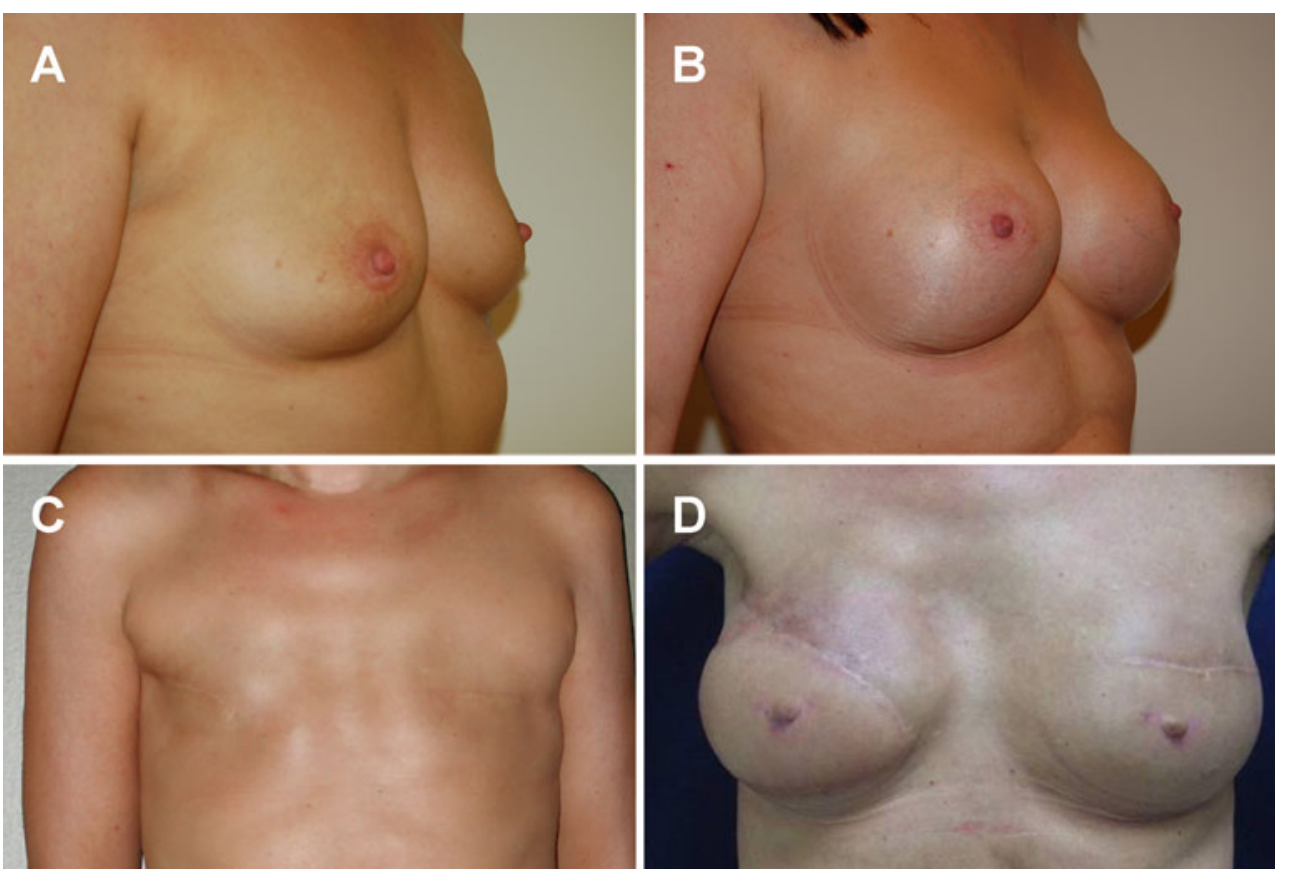

contracture. The latter phenomenon is only seen sporadically in patients who had SG implants before they received the $\mathrm{MH}$ implant. The $\mathrm{MH}$ implant is reported by patients to take on body temperature easier than alternative implants, and radiologists have less difficulty in examining the mammary gland. It is delivered in many sizes as a round implant with a high profile and has a CE mark. There is a chance of visible rippling when the skin and subcutaneous cover are thin. This can be corrected with lipofilling or the use of a commercial Tissue Matrix.

There are two indications for using the MH implant. The first is the choice of women who do not want the SG implant nor an extensive autologous reconstruction, and the second, to have another option for women who have reacted adversely to the SG implant.

In summary, the main characteristics of the $\mathrm{MH}$ implant are the possession of a CE mark, a biodegradable filling, self-alarm and easy replacement under local anaesthesia in an outpatient procedure in case of a spontaneous rupture, a natural look and feel, and lack of capsular contracture. In this article, the performance of the $\mathrm{MH}$ implant is presented separately from that of the $\mathrm{SG}$ implants. The $\mathrm{MH}$ implant should not be confused with the related Poly Implant Prostheses (PIP) implant, which has a content of hydroxypropyl cellulose hydrogel. The PIP implant was taken from the market by the Medical Devices Agency in the UK because there was one report of a filler leak and the manufacturer's biological safety assessment of this product was inadequate. Explantation of the PIP implant was not recommended. The aim of this study was to investigate the behaviour of the $\mathrm{MH}$ implant and to investigate whether women with $\mathrm{MH}$ implants may inciden- tally experience similar health complaints as those that are sometimes seen in women with SG implants.

\section{Methods}

Women in a general plastic surgery practice who received the $\mathrm{MH}$ implant either as the first implant ( $\mathrm{C}$ group, $n=42$ ) or had a replacement of the $\mathrm{SG}$ implant by the $\mathrm{MH}$ implant because of health complaints (B group, $n=35$ ) filled out questionnaires with regard to complaints that patients with SG implants in some cases tend to have, such as chronic fatigue, painful joints and muscles, etc. Participants could also give their opinion on the aesthetic results. The B group was subdivided in those who had an additional capsulectomy (B1 group, $n=22$ ) and those without capsulectomy (B2 group, $n=13$ ). The same questions were put forward to the control group $(n=34)$, which was recruited from healthy female hospital workers without breast implants. The reader is referred to Table 1 for an overview of the general characteristics of the groups. When the results for pre- and post-operative parameters were compared, paired $t$ tests were used to analyse the statistical significance.

\section{Results}

Women with $\mathrm{MH}$ breast implants (C group), SG implants replaced by $\mathrm{MH}$ implants (B1 and B2 groups), and a control group without implants were asked to share their experiences through filling out questionnaires focused on health problems and general aspects related to the breast 
Table 1 Overview of all participating groups

\begin{tabular}{|c|c|c|c|c|c|c|c|}
\hline Group & $\begin{array}{l}\text { Number of } \\
\text { patients }\end{array}$ & $\begin{array}{l}\text { Mean age } \\
\text { (years) }\end{array}$ & $\begin{array}{l}\text { Mean duration SG } \\
\text { implant (years) }\end{array}$ & Capsulectomy & $\begin{array}{l}\text { Mean follow-up } \\
\text { (years) }^{\mathrm{a}}\end{array}$ & $\begin{array}{l}\text { SG implant } \\
\text { rupture (\%) }\end{array}$ & $\begin{array}{l}\text { Pre-operative } \\
\text { complaints (\%) }\end{array}$ \\
\hline Control & 34 & 35.0 & n.a. & n.a. & n.a. & n.a. & $10(29)$ \\
\hline B1 & 22 & 50.5 & 15.5 & yes & 3 & $12(55)$ & $18(82)$ \\
\hline B2 & 13 & 47.5 & 10.5 & no & 3.5 & $5(39)$ & $10(77)$ \\
\hline $\mathrm{C}$ & 42 & 36.0 & 0 & n.a. & 3 & n.a. & $6(14)$ \\
\hline
\end{tabular}

n.a. not applicable

${ }^{\text {a }}$ Postoperative follow-up period

augmentation or reconstruction. The questionnaires were filled out on average 3-3.5 years after the operation during which the implant was inserted/replaced. Prior to the replacement, women of the B groups had SG implants for an average period of 13 years. The control group had hardly
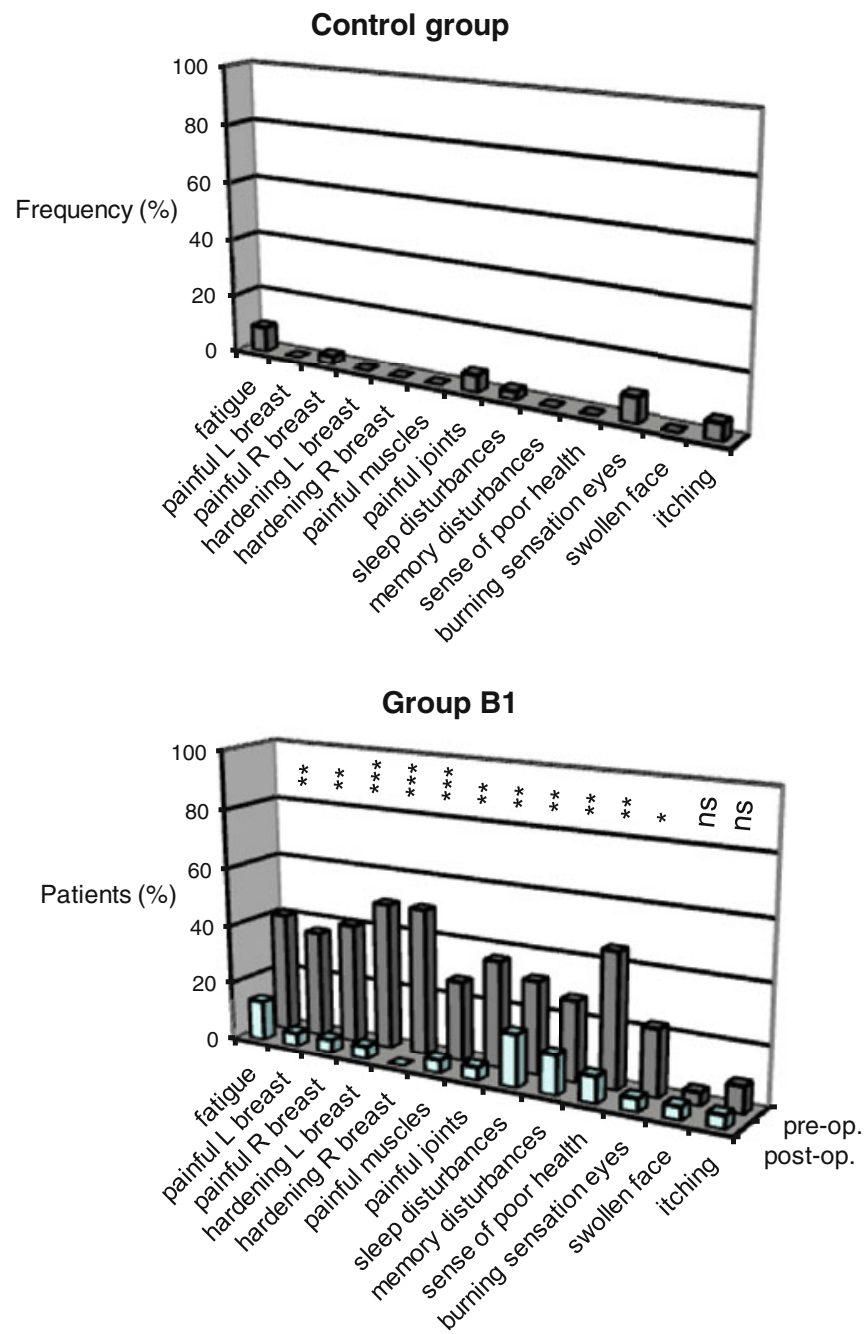

Fig. 2 Complaints reported by patients and controls. The frequency of complaints (specified below the graphs) reported by the different patient groups before (pre-op.) and after (post-op.) $\mathrm{MH}$ implant insertion ( $C$ group) or replacement of their breast implants ( $B 1$ and $B 2$ any complaints, nor did the women of the $\mathrm{C}$ group. With regard to their pre-operative health complaints, the women of the B group improved on the whole spectrum, which was statistically significant for most complaints. Importantly, the improvement appeared to be more pronounced when a
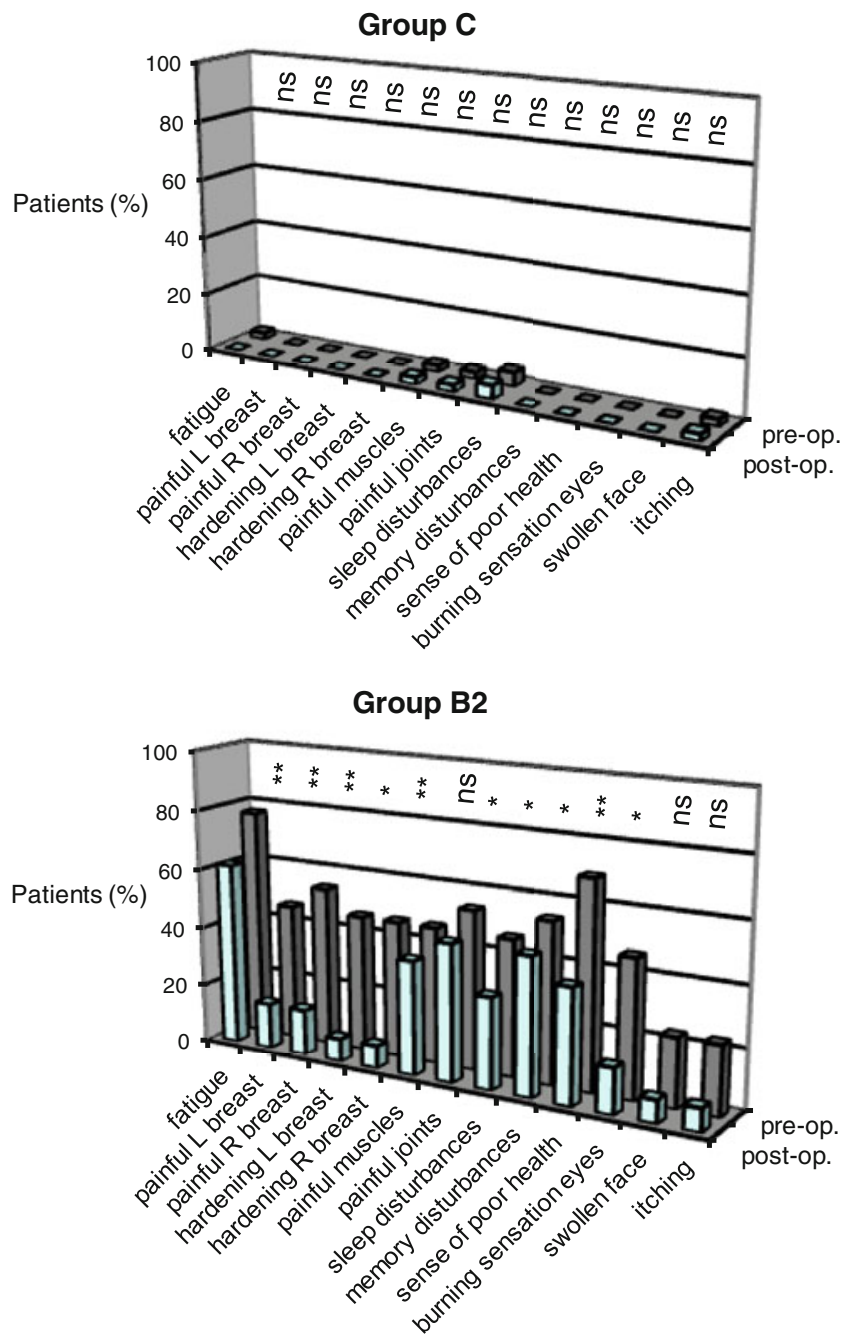

groups), and those reported by the Control group without implants is illustrated by the graphs. The results were analysed by paired $t$ tests: $n s$ not significant, $* p<0.05 ; * * p<0.01 ; * * * p<0.001$ 
Fig. 3 Patients' opinion on the results of breast augmentation/ reconstruction with $\mathrm{MH}$ implants. The frequency by which patients experienced changes is graphically illustrated. Improvements are indicated by the green bars, no change by the yellow bars and a deterioration by the red bars. The parameters evaluated as a result of $\mathrm{MH}$ implantation ( $C$ group) or SG implant replacement with $\mathrm{MH}$ implants (B1 and $B 2$ groups) are specified below the graph

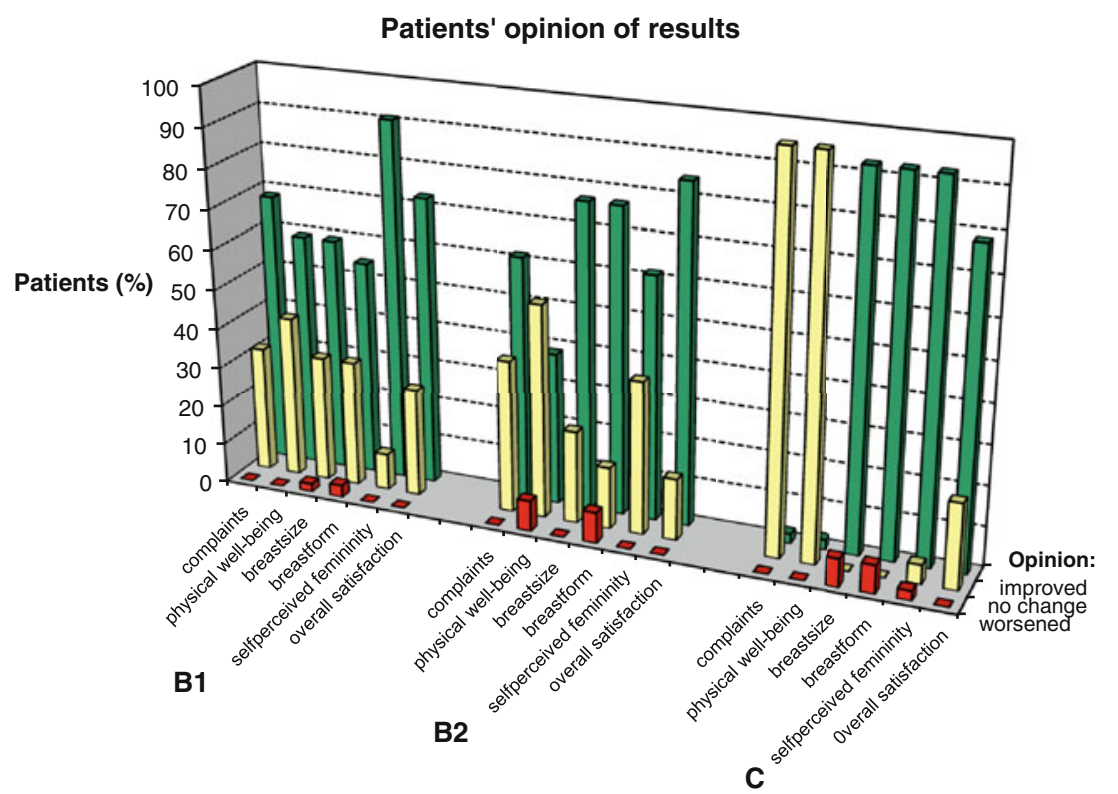

capsulectomy was also performed (Fig. 2). In both the B and the $\mathrm{C}$ groups, the satisfaction of women with the $\mathrm{MH}$ implant was high (Fig. 3).

\section{Discussion}

Working with the $\mathrm{MH}$ implant and keeping an eye on the complaints that over the years have been mentioned by women with the SG implant [2-8], it became obvious that there might be a novel disease that is probably caused by silicone gel bleed [9-15]. The major difference between the $\mathrm{MH}$ and $\mathrm{SG}$ implants is the content of the implant. Both have a silicone elastomere shell, which is a solid rubber. Since patients who start on the $\mathrm{MH}$ implant do not develop the same complaints as some patients with the SG implant, even after a longer follow-up period than was used in this study for the $\mathrm{B}$ and the $\mathrm{C}$ groups (unpublished results), it can be concluded that the silicone elastomere shell does not seem to play a role in development of complaints. Moreover, the replacement of the SG implant by an $\mathrm{MH}$ implant resulted in a pronounced reduction of complaints, which suggests a direct connection between the contents of the SG implant and the complaints. This connection is seen in the B group for ruptured as well as intact SG implants (data not shown), indicating that bleeding rather than rupture of the implants is associated with the appearance of complaints. This is also in concurrence with the finding that a capsulectomy (B1 group) resulted in a stronger reduction of complaints. The explanation could be that the capsule is the first line of defence of the body against gel bleed [11, 16] and will absorb the bleed until it can no longer be contained. If the capsule is left in place during the replacement operation, it will gradually regress, leaving the bled molecules behind $[2,9,12,14,15]$ and loosing the supreme moment to gain clearance of much of the bleed in women with complaints. Our data suggest that a simultaneous capsulectomy in patients with complaints is preferred for an optimal reduction of these complaints. However, in certain B group patients, a number of complaints remained, even after capsulectomy. It is possible that in these patients, a certain threshold has been passed and that a chronic disease has been generated that might have been triggered by the exposure to silicones, but is not dependent on this anymore. Indeed, symptoms of chronic autoimmune diseases have been frequently reported in patients with SG implants [4, 6, 8]. A larger retrospective study focused on a comparison of the $\mathrm{MH}$ and $\mathrm{SG}$ implants has been performed and is in preparation. The drawbacks of the $\mathrm{MH}$ implant can be easily overcome. For instance, rippling in case of a thin soft tissue cover can be corrected by lipofilling or insertion of a Tissue Matrix. Surgeons tend to be taken aback by the innocuous clear serous fluid in case of rupture of the $\mathrm{MH}$ implant (Fig. 4), but one should keep in mind that rupture of an SG implant goes unnoticed by the patient and will lead to a highly increased exposure to silicones, which may be detrimental to a women's health [5]. Moreover, the insidious gel bleed of an intact SG implant over the years can be catastrophic in some women, who are genetically predisposed to the development of chronic health complaints in relation to the SG implant, even though the pathogenetic mechanism remains elusive. 



Fig. 4 Replacement of ruptured MH implant. A rupture of the MH implant on the right side caused an enlargement of the breast within a couple of days (a). The scar and capsule are incised under local anaesthesia and the clear serous fluid is drained. The ruptured implant is removed easily and the pocket is rinsed (b). A new MH implant is inserted without drains, resulting in a normal breast size immediately after the short procedure (c). Directly following this procedure, the treated woman has to wear a supporting and compressive bra for a period of 3 weeks

\section{Conclusion}

For women who do not wish to have an SG implant either for augmentation or reconstruction, or who have developed health complaints in relation to SG implants, or those who do not want to have an extensive autologous breast reconstruction with additional scarring, the $\mathrm{MH}$ implant appears to be a good option. One should realise though that it has to be replaced now and then because it is not everlasting. But neither is the SG implant. Once a woman is informed about the advantages and disadvantages of the MH and SG implants, she can give a truly informed consent about either implant and then she seems to be in favour of the $\mathrm{MH}$ implant. Our study demonstrates that the vast majority of individuals are satisfied with the MH implant.

Open Access This article is distributed under the terms of the Creative Commons Attribution Noncommercial License which permits any noncommercial use, distribution, and reproduction in any medium, provided the original author(s) and source are credited.

\section{References}

1. Arion H (2001) Carboxy-methyl cellulose hydrogels used to fill breast implants: 15 years of experience. Eur J Plast Surg 24:172-175

2. Ahn CY (1994) Regional silicone-gel migration in patients with ruptured implants. Ann Plast Surg 33:201-208

3. Vasey FB, Zarabadi SA, Seleznick M, Ricca L (2003) Where there's smoke there's fire: the silicone breast implant controversy continues to flicker: a new disease that needs to be defined. J Rheumatol 30:2092-2094

4. Bridges AJ, Conley C, Wang G, Burns DE, Vasey FB (1993) A clinical and immunologic evaluation of women with silicone breast implants and symptoms of rheumatic disease. Ann Intern Med 118:929-936

5. Vermeulen RC, Scholte HR (2003) Rupture of silicone gel breast implants and symptoms of pain and fatigue. J Rheumatol 30:2263-2267

6. Shanklin DR, Smalley DL (1998) The immunopathology of siliconosis. Immunol Res 18:125-173

7. Solomon G (1994) A clinical and laboratory profile of symptomatic women with silicone breast implants. Sem Arthr Rheum 24:29-37

8. Selva-O'Callaghan A, Tura JM, Grau-Junyent JM, LabradorHorrillo M, Solans-Laque R, Vilardell-Rarrés M (2004) Silicone gel filled breast implants and dermatomyositis. Clin Exp Rheumatol 22:376

9. Barker DE, Retsky MI, Schulz S (1978) "Bleeding" of silicone from bag-gel breast implants, and its clinical relation to fibrous capsule reaction. Plast Reconstr Surg 61:836-841

10. Hardt NS, Emery JA, Steinbach BG, La Torre G, Caffee H (1995) Cellular transport of silicone from breast prostheses. Int J Occup Med Tox 4:127-134

11. BeekmanWH FR, Van Diest PJ, Hage JJ (1997) Migration of silicone through the fibrous capsules of mammary prostheses. Ann Plast Surg 38:441-445

12. Garrido L, Pfleiderer B, Jenkins BG, Hulka CA, Kopans DB (1994) Migration and chemical modification of silicone in women with breast prostheses. Magn Reson Med 31:328-330

13. Smahel J (1979) Foreign material in the capsules around breast prostheses and the cellular reaction to it. Br J Plast Surg 32:35-42

14. Holten IW, Barnett RA (1995) Intraductal migration of silicone from intact gel breast prostheses. Plast Reconstr Surg 95:563-566

15. Truong LD, Cartwright J, Goodman MD, Woznicki D (1988) Silicone lymphadenopathy associated with augmentation mammaplasty, morphologic features of nine cases. Am J Surg Pathol 12:484-491

16. Peters W, Smith D, Lugowski S (1996) Failure properties of 352 explanted silicone-gel breast implants. Can J Plast Surg 4:55-58 